\title{
Berberine induces selective apoptosis through the AMPK-mediated mitochondrial/caspase pathway in hepatocellular carcinoma
}

\author{
XIAOLONG YANG and NING HUANG \\ Research Unit of Infection and Immunity, Department of Pathophysiology, West China School of \\ Preclinical and Forensic Medicine, Sichuan University, Chengdu, Sichuan 610041, P.R. China
}

Received December 25, 2012; Accepted April 23, 2013

DOI: $10.3892 / \mathrm{mmr} .2013 .1506$

\begin{abstract}
Advanced hepatocellular carcinoma (HCC) is insensitive to traditional chemotherapeutic approaches, which causes difficulty in the development of novel agents for the treatment of HCC. Berberine is the main component of Coptidis Rhizoma, a plant alkaloid with a long history of use in Chinese medicine, and has become a potential candidate for the treatment of HCC due to its high antitumor activity and low toxicity. In this study, we investigated the mechanism via which berberine exerts its inhibitory effects on HCC. The data demonstrated that berberine selectively decreased cell viability in a time- and dose-dependent manner in the HepG2, SMMC-7721 and Bel-7402 HCC cell lines compared with normal hepatocytes (HL-7702 cells), as determined by a sulforhodamine B assay. Flow cytometric analysis revealed that berberine increased the number of late apoptotic cells. Pretreatment with berberine in HepG2 cells resulted in a significant increase in phosphorylated AMP-activated protein kinase (AMPK), as well as a marked elevation in phosphorylated Akt levels. In addition, the activation of AMPK was accompanied by apoptotic effects that occurred in a caspase-dependent manner through the mitochondrial pathway, and was coupled with the release of cytochrome $\mathrm{c}$ from the mitochondria and the activation of caspase-9 and -3. Furthermore, the ratio of Bax/Bcl-2 was increased in a dose-dependent manner. Our study supports the theory that berberine selectively inhibits the growth of human hepatocellular cancer cells by inducing AMPK-mediated caspase-dependent mitochondrial pathway cell apoptosis, and rarely causes cytotoxicity in normal cells. Therefore, berberine is a promising novel agent for the treatment of HCC.
\end{abstract}

Correspondence to: Professor Ning Huang, West China School of Preclinical and Forensic Medicine, Sichuan University, 17\#, Section 3, Ren Min Nan Lu, Chengdu, Sichuan 610041, P.R. China

E-mail: huangpanxiao@sina.com

Key words: hepatocellular carcinoma, berberine, selective, AMP-activated protein kinase, apoptosis

\section{Introduction}

Hepatocellular carcinoma (HCC) is one of the most common and aggressive malignant tumor types worldwide. The incidence of HCC has markedly increased to $>600,000$ cases annually, and HCC has become the second most common cause of mortality in China $(1,2)$. Numerous risk factors, including chronic infection with hepatitis $\mathrm{B}$ and $\mathrm{C}$ viruses, alcohol-related cirrhosis and non-alcoholic fatty liver diseases, are closely correlated with the occurrence and development of HCC (3-5). Furthermore, a large percentage of patients with advanced HCC require systemic or selective chemotherapy to achieve a longer life expectancy. Unfortunately, the overall survival rate remains poor with the use of traditional chemotherapy, as HCC is insensitive to conventional cytotoxic agents $(6,7)$. Therefore, there is a significant requirement for the development of innovative treatment approaches to improve patient outcome.

AMP-activated protein kinase (AMPK) is a metabolic sensing protein kinase that acts as an important energy sensor, mainly in ATP-deprived conditions (8). Therefore, AMPK is known to play a major protective role under conditions of metabolic stress. In the activated state, AMPK downregulates several anabolic enzymes and thus turns off ATP-consuming metabolic pathways $(9,10)$. Several studies have observed the strong pro-apoptotic potential of AMPK in activated conditions, including AMPK activator (AICAR)-treated cells or constitutively active AMPK mutants (11). Collectively, these studies highlight the importance of AMPK as a direct therapeutic target in all stages of cancer.

Berberine, an alkaloid purified from the Berberis species (Fig. 1), has been extensively studied and is known to exhibit multiple pharmacological activities, including antiprotozoal, antihypertensive, antibacterial, anti-inflammatory, anticholinergic and anti-arrhythmic effects (12-14). Previous studies have demonstrated that berberine has anticancer activities against several types of cancer, to include liver cancer $(15,16)$. Berberine has been demonstrated to induce apoptosis in numerous cultured cancer cell lines $(17,18)$; however, the molecular mechanisms underlying berberine-induced apoptosis and the pathways involved have not been fully elucidated to date. In the present study, we aimed to inves- 
tigate the inhibitory effect of berberine on HCC cell lines and determine whether berberine-induced apoptosis was correlated with the AMPK-mediated caspase-dependent mitochondrial pathway.

\section{Materials and methods}

Reagents. Berberine (purity, >99\%), sulforhodamine B (SRB) and protease inhibitors were purchased from Sigma-Aldrich (St. Louis, MO, USA). The high-glucose medium, Dulbecco's Modified Eagle's Medium (DMEM), was obtained from Gibco-BRL (Carlsbad, CA, USA). The Bradford Protein Assay kit, RIPA lysis buffer and the Annexin V-FITC Apoptosis Detection kit were purchased from Beyotime Institute of Biotechnology (Jiangsu, China). Activated signaling pathways were identified using primary antibodies, including anti-AMPK, anti-phosphospecific AMPK, anti-Akt, anti-phosphospecific Akt, anti-Bax, anti-Bcl-2, anti-cytochrome c, anti-caspase-3 and anti-caspase-9, purchased from Santa Cruz Biotechnology, Inc. (Santa Cruz, CA, USA). The internal control antibody (anti- $\beta$-actin) was also obtained from Santa Cruz Biotechnology, Inc.

Cell culture. The human hepatocelluar carcinoma cell lines (HepG2, SMMC-7721 and Bel-7402) and the normal liver cell line (HL-7702) were purchased from Hsiang-Ya Medical College (Hunan, China). Cells were cultured at $37^{\circ} \mathrm{C}$ and $5 \% \mathrm{CO}_{2}$ in DMEM supplemented with $10 \%$ fetal bovine serum (FBS), penicillin $(100 \mathrm{U} / \mathrm{ml})$ and streptomycin $(100 \mathrm{mg} / \mathrm{ml})$. The study was approved by the ethics committee of Sichuan University.

Cell treatment and cell viability assay. The in vitro cytotoxicity of berberine against HepG2, SMMC-7721, Bel-7402 and HL-7702 cells was assessed using the traditional SRB assay. The SRB assay is routinely used for cytotoxicity determination, based on the measurement of live cell protein content (19). In brief, cells were seeded into 96-well plates at a density of 7,000 cells/well. Following $24 \mathrm{~h}$ of culture in DMEM supplemented with $10 \%$ FBS, the medium was replaced with DMEM without FBS for $24 \mathrm{~h}$. Subsequently, the cells were treated with berberine at final concentrations of $3.125,6.25,12.5,25,50$ and $100 \mu \mathrm{M}$ for 24 or $48 \mathrm{~h}$ prior to the SRB assay. All experiments were conducted in parallel with controls; at 24 or $48 \mathrm{~h}, 100 \mu \mathrm{l}$ of $10 \%$ trichloroacetic acid was also added to each well when staining for $30 \mathrm{~min}$, and then the excess dye was removed by washing repeatedly with $1 \%$ acetic acid. The protein-bound dye was dissolved in $10 \mathrm{mM}$ Tris Base solution (Santa Cruz Biotechnology, Inc.) for optical density (OD) determination at $570 \mathrm{~nm}$ using a microculture reader (Bio-Rad, Richmond, CA, USA). The percentage of viable cells was calculated as follows: (A of experimental group/A of control group) x100, where A represents the absorbance.

Apoptosis assays by flow cytometry (FCM). Apoptosis was analyzed by FCM using the Annexin V-FITC Apoptosis Detection kit (Beyotime Institute of Biotechnology), according to the manufacturer's instructions. Briefly, following treatment with $0,12.5,50$ and $100 \mu \mathrm{M}$ berberine for $24 \mathrm{~h}, \mathrm{HepG} 2$ cells

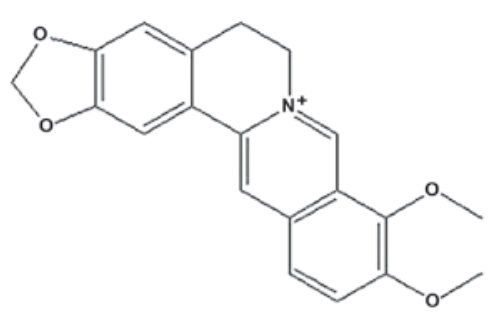

Figure 1. Structure of berberine.

were detached and resuspended in $100 \mu 1$ binding buffer containing fluorescein isothiocyanate-conjugated annexin $\mathrm{V}$ and propidium iodide (PI). Following incubation for $15 \mathrm{~min}$ at room temperature in the dark, the cells were analyzed by FCM (BD Biosciences, Franklin Lakes, NJ, USA).

Western blot assay. The cells were washed twice with cold phosphate-buffered saline (PBS) and then suspended in RIPA buffer for $30 \mathrm{~min}$ on ice. The supernatant was collected as the total protein extract. The protein concentration was estimated by the Bradford Protein Assay kit (Beyotime Institute of Biotechnology), according to the manufacturer's instructions. Equal amounts of protein $(40 \mu \mathrm{g})$ were analyzed by sodium dodecyl sulphate-polyacrylamide gel electrophoresis (SDS-PAGE) with a 5 or $12 \%$ separation gel, and molecular weight markers were run simultaneously. The proteins were transferred to nitrocellulose membranes and then blocked for $2 \mathrm{~h}$ at room temperature in a solution of $5 \%$ milk or bovine serum albumin (BSA). Incubation with the appropriate primary antibodies was performed overnight at $4^{\circ} \mathrm{C}$. Membranes were incubated with horseradish peroxidase (HRP)-conjugated goat anti-rabbit or anti-mouse secondary antibody (1:2,000 dilution) for $2 \mathrm{~h}$ at room temperature with gentle agitation. After washing, bands were visualized by an Enhanced Chemiluminescence (ECL) Western Blotting Detection system (Amersham Pharmacia Biotech, NJ, USA).

Statistical analysis. All data were expressed as the mean \pm SE. The statistical significance of differences in the data was determined by Student's t-test. $\mathrm{P}<0.05$ was considered to indicate a statistically significant difference.

\section{Results}

Berberine inhibits cell viability in HCC cell lines in a time- and dose-dependent manner. To evaluate the antitumor characteristics of berberine, cell viability was measured by a standard SRB assay following brief exposure to berberine ( 24 or $48 \mathrm{~h}$ ). As demonstrated in Fig. 2, berberine significantly reduced cell viability in human hepatoma cell lines in a time- and dose-dependent manner. Subsequently, we predicted the side effects of berberine by exposing the normal hepatic cell line, HL-7702, to the agent. The $\mathrm{IC}_{50}$ values in the HL-7702 cell line $(838.4 \mu \mathrm{M})$ at $48 \mathrm{~h}$ were markedly higher than those of the HCC cell lines $(34.5 \mu \mathrm{M}, 25.2 \mu \mathrm{M}$ and $53.6 \mu \mathrm{M}$ in the HepG2, SMMC-7721 and Bel-7402 cell lines, respectively). The differences in the $\mathrm{IC}_{50}$ values indicated that berberine was able to selectively decrease the cell viability of HCC cells compared with normal hepatocytes. 
A

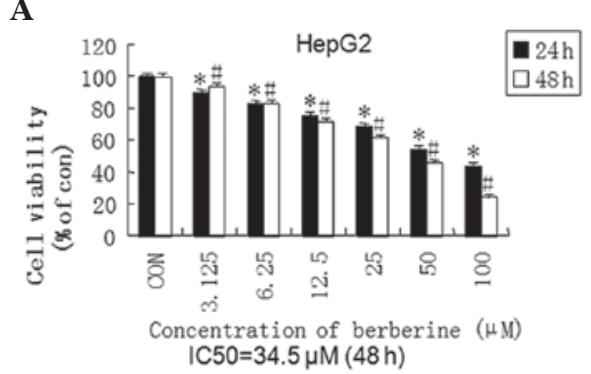

C

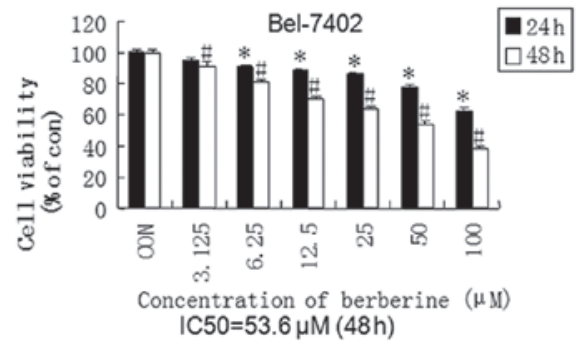

B

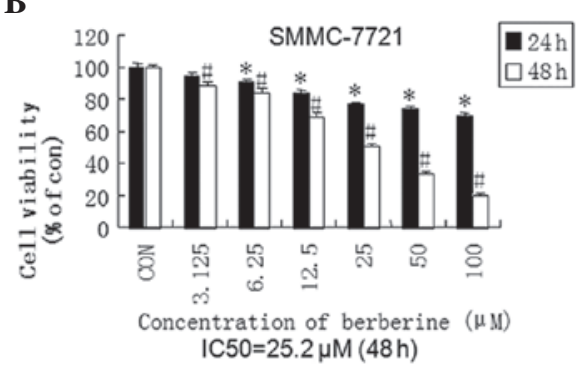

D

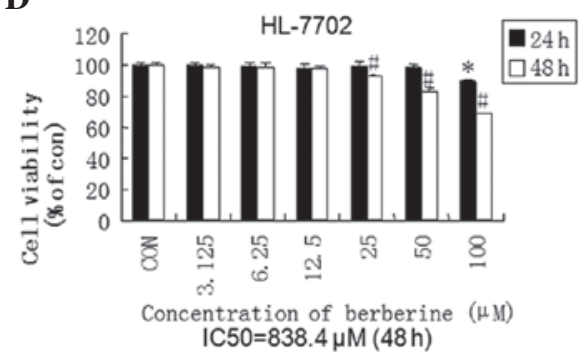

Figure 2. Berberine induces the growth inhibition of human hepatocytes. All three hepatocellular carcinoma (HCC) cell lines (HepG2, SMMC-7721 and Bel-7402) and normal HL-7702 human hepatic cells were treated with increasing concentrations of berberine (3.125, 6.25, 12.5, 25, 50 and 100 $\mu \mathrm{M})$, and cell viability was analyzed by a sulforhodamine B (SRB) assay. Data are presented as the percentage of untreated controls. ${ }^{*} \mathrm{P}<0.05$ and ${ }^{\#} \mathrm{P}<0.05$ vs. the controls.

Berberine increases apoptosis in HepG2 cells. To further investigate the inhibition mechanism of berberine in $\mathrm{HCC}$, we selected HepG2 cells for further study. HepG2 cells were treated with $12.5,25$ and $50 \mu \mathrm{M}$ berberine for $24 \mathrm{~h}$, and apoptosis was detected by flow cytometric analysis with the Annexin V-FITC Apoptotic Detection kit (Beyotime Institute of Biotechnology). Data revealed that the pretreatment of HepG2 cells with berberine significantly increased the number of early apoptotic cells (annexin $\mathrm{V}^{+}$and $\mathrm{PI}^{-}$) and late apoptotic cells (annexin $\mathrm{V}^{+}$and $\mathrm{PI}^{+}$) compared with the control group, in a dose-dependent manner (Fig. 3A). At a concentration of $50 \mu \mathrm{M}$, berberine led to $\leq 40 \%$ apoptosis in HepG2 cells (Fig. 3B). These results suggested that berberine-induced apoptosis in the liver cancer cells contributed to its antiproliferative and cytotoxic effects.

Berberine induces apoptosis via the mitochondrial-dependent pathway. In chemically induced apoptosis, mitochondria play a central role in the commitment of cells to apoptosis through cytochrome c-dependent pathways. To elucidate the molecular mechanism of berberine-induced apoptosis in HCC cells, we examined the expression of proteins associated with apoptosis. HepG2 cells were treated with 12.5 and $50 \mu \mathrm{M}$ berberine for $24 \mathrm{~h}$. The results demonstrated that creatine kinase induces the release of cytochrome $\mathrm{c}$ from mitochondria to the cytosol in dose-dependent manner (data not shown). Caspase- 9 and -3 , well-known downstream molecules of cytochrome c, were also cleaved in a concentration-dependent manner (Fig. 4A). We further evaluated the effect of berberine on the mitochondrial apoptosis signaling pathway. We found that the expression of $\mathrm{Bcl}-2$ protein, an anti-apoptotic molecule, decreased in a dose-dependent manner, whereas the expression of the pro-apoptotic protein, Bax, was significantly increased (Fig. 4B). Furthermore, the ratio of Bax/Bcl-2 was increased in a dose-dependent manner (Fig. 4C). These results indicated that treatment with berberine leads to a shift
A
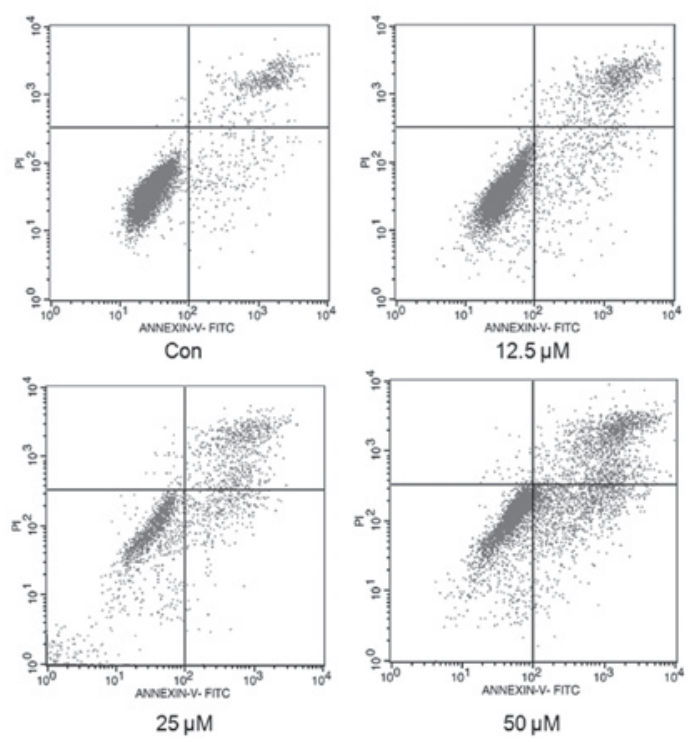

B

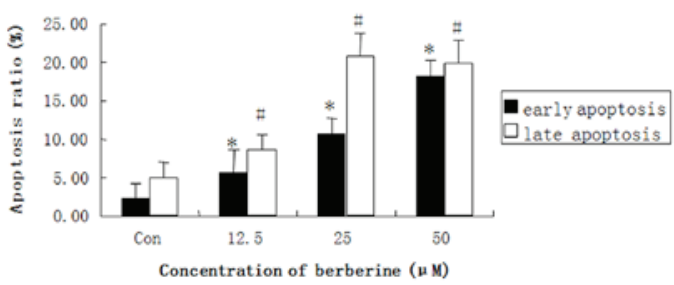

Figure 3. Berberine induces apoptosis in the HepG2 hepatocellular carcinoma (HCC) cell line. (A) Flow cytometric analysis with annexin V-FITC/propidium iodide (PI) double staining. Cells were incubated in the absence (Con) or presence of berberine (12.5, 50 and $100 \mu \mathrm{M})$ for $24 \mathrm{~h}$. Normal cells stained negative for annexin V-FITC and PI (bottom left quadrant). Following incubation with berberine for 24 h, early apoptotic cells stained positive for annexin V-FITC and negative for PI (bottom right quadrant), while late apoptotic cells stained positive for annexin V-FITC and PI (top right quadrant). (B) The histogram represents the sum of early and late apoptotic cells induced by $12.5,50$ and $100 \mu \mathrm{M}$ berberine treatment for $24 \mathrm{~h}$. Data are presented as the mean \pm SE from triplicate results and three independent experiments. ${ }^{*} \mathrm{P}<0.05$ and ${ }^{\#} \mathrm{P}<0.05$ vs. the controls. 
A

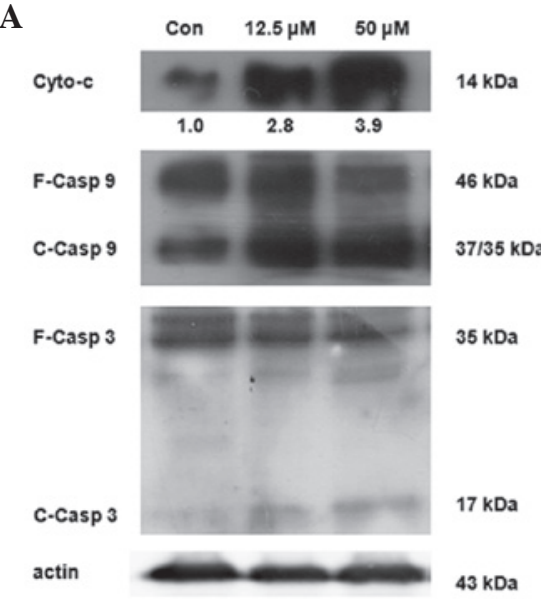

B

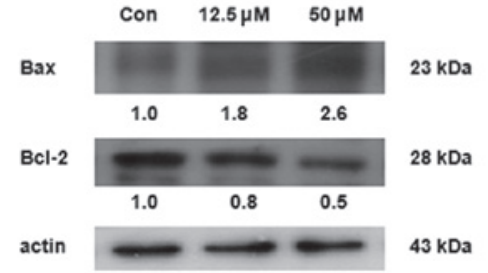

C
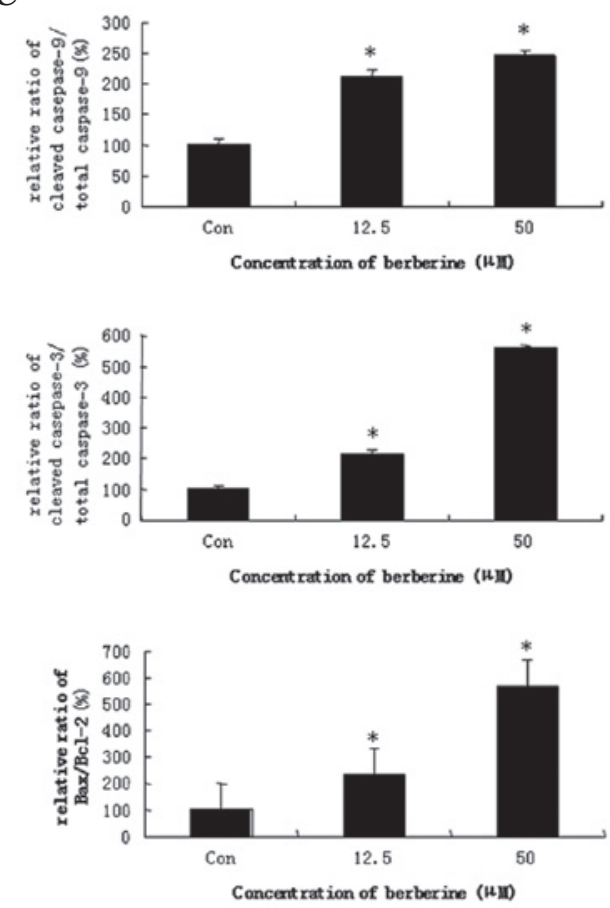

Figure 4. Berberine induces caspase-dependent apoptosis in the HepG2 hepatocellular carcinoma (HCC) cell line. HepG2 cells were treated with 12.5 and $50 \mu \mathrm{M}$ berberine for $24 \mathrm{~h}$. (A) The protein expression of cytochrome c and caspase- 9 and -3 in HepG2 cells was analyzed by western blot analysis. (B) The protein expression of Bax and Bcl-2 in HepG2 cells was analyzed by western blot analysis. The numbers below the bands indicate the relative density ratio of each protein normalized by the internal control ( $\beta$-actin). The experiments were performed in triplicate. (C) The ratios of cleaved-caspase-9/total caspase- 9 , cleaved-caspase-3/total caspase- 3 and $\mathrm{Bax} / \mathrm{Bcl}-2$ are presented as the mean $\pm \mathrm{SE}(\mathrm{n}=3)$. ${ }^{*} \mathrm{P}<0.05$ vs. the controls.

A

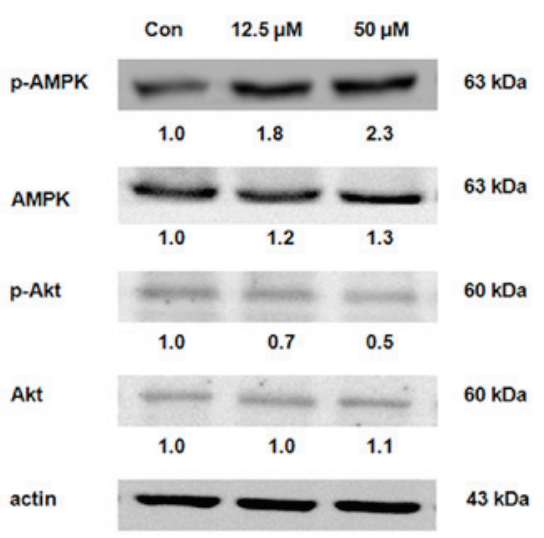

B
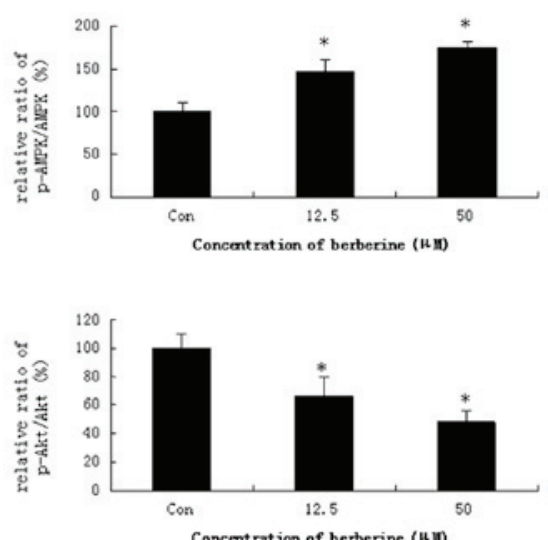

Figure 5. Protein expression of AMP-activated protein kinase (AMPK), phosphorylated (p)-AMPK, Akt and p-Akt in HepG2 cells treated with the same dose of berberine and analyzed by western blot analysis. (A) The numbers below the bands indicate the relative density ratio of each protein normalized by the internal control ( $\beta$-actin). The experiments were performed in triplicate. (B) The ratios of p-AMPK/AMPK and p-Akt/Akt are presented as the mean \pm SE $(\mathrm{n}=3) .{ }^{*} \mathrm{P}<0.05$ vs. the controls.

from anti-apoptosis to pro-apoptosis by altering the function of the proteins in the Bcl-2 family, which results in the release of cytochrome $\mathrm{c}$ from mitochondria, thus inducing caspase-dependent mitochondrial pathway cell apoptosis.

Effects of berberine on the protein expression of AMPK and Akt. Several reports have demonstrated that activation of AMPK leads to the induction of apoptosis in numerous human cancer cell types $(20,21)$. Therefore, we investigated whether the phosphorylation of AMPK is induced by berberine. HepG2 cells were treated with 12.5 and $50 \mu \mathrm{M}$ berberine for $24 \mathrm{~h}$. As demonstrated in Fig. 5A, compared with the basal level, berberine significantly stimulated the phosphorylation of AMPK (Thr-172), which increased the ratio of p-AMPK/total-AMPK in dose-dependent manner. The measurements of the phosphorylation pattern of Akt revealed that the pretreatment of HepG2 cells with berberine resulted in a marked elevation in the levels of phosphorylated Akt (Ser-473) relative to the control cells, without significantly altering the total protein levels of Akt (Fig. 5B). These results 
indicated that AMPK activation is correlated with the induction of apoptosis.

\section{Discussion}

$\mathrm{HCC}$ has a higher prevalence and mortality rate compared with other types of cancer. Therefore, prolonging survival times and improving quality of life have become main objectives in the treatment and management of patients with advanced HCC. The antitumor property of berberine has recently attracted increasing research attention (22). A crucial advantage for the use of natural products as an alternative to the chemotherapeutic approach is their low cytotoxicity. In the present study, we examined the effect of berberine on cell viability and apoptosis in normal and cancer cells. We observed that berberine suppressed cell growth in a dose- and time-dependent manner in human HepG2, SMMC-7721 and Bel-7404 HCC cell lines. This indicates that the inhibitory effect of berberine in liver cancer is non-species-specific, and is associated with the previously reported types of HCC cells (23). The normal control liver cell line, HL-7702, exhibited decreased cell viability.

Berberine is one of the key components of Coptis chinesis, which is frequently utilized in proprietary Chinese herbal drugs and exerts a wide range of pharmacological effects. Berberine exhibits high antitumor activity in various types of tumors through different mechanisms $(16,17,24)$. A number of factors, including cytokines, chemokines, receptors and downstream elements within signaling cascade pathways, are correlated with apoptosis induced by berberine. Berberine has been demonstrated to induce apoptosis in human HCC cells via Fas-mediated (15) inhibition of the mTOR-signaling pathway (16), downregulation of MMP-9 expression (25) and the nuclear factor- $\kappa \mathrm{B}(\mathrm{NF}-\kappa \mathrm{B})$ and activator protein 1 (AP-1) pathways (26). In the current study, we demonstrated that berberine had cytotoxic effects in HepG2 cells, including annexin $\mathrm{V}$ binding, cytochrome $\mathrm{c}$ release and activation of caspases, indicated by the increased cleavage of caspase- 9 and -3 , via caspase-dependent mitochondrial pathway cell apoptosis.

The association between cancer and AMPK emerges as an intriguing area of investigation. Numerous studies have indicated that the AMPK pathway is implicated in cancer. While traditionally regarded as a sensor of cellular energy status and a regulator of metabolism, AMPK has been linked to tumor suppressors, including LKB1, p53, TSC1 and TSC2, providing novel support for the theory that AMPK may function as a suppressor of cell proliferation (27). There has been much speculation on the role of AMPK in cancer, as this pathway may be either anti-apoptotic or pro-apoptotic depending on the conditions (28). The results of the present study demonstrated that treatment with berberine promoted AMPK phosphorylation and inhibited Akt phosphorylation in HepG2 cells, which led to caspase-dependent mitochondrial pathway cell apoptosis.

In conclusion, to the best of our knowledge, our results demonstrate for the first time that berberine exerts a selective antitumor effect on human liver cancer cells when compared with non-tumorigenic HL-7702 normal liver cells. Additionally, our data provide support to the theory that
AMPK may be involved in the antitumor effect of berberine via caspase-dependent mitochondrial pathway cell apoptosis. These findings provide a molecular basis for the antiproliferative activity of berberine, which may be used as a potent and alternative chemotherapeutic agent for the treatment of HCC.

\section{Acknowledgements}

This study was supported by the China National Nature Science Fund (no. 30671963), the China Medical Board of New York Inc. (no. 98-681) and the Sichuan University 985 project (Science and Technology Innovation Platform for Novel Drug Development).

\section{References}

1. Parkin DM, Bray F, Ferlay J and Pisani P: Global cancer statistics, 2002. CA Cancer J Clin 55: 74-108, 2005

2. Tang ZY, Ye SL, Liu YK, et al: A decade's studies on metastasis of hepatocellular carcinoma. J Cancer Res Clin Oncol 130: $187-196,2004$

3. Ming L, Thorgeirsson SS, Gail MH, et al: Dominant role of hepatitis B virus and cofactor role of aflatoxin in hepatocarcinogenesis in Qidong, China. Hepatology 36: 1214-1220, 2002.

4. El-Serag HB: Epidemiology of hepatocellular carcinoma in USA. Hepatol Res 37 (Suppl 2): S88-S94, 2007.

5. Parkin DM: The global health burden of infection-associated cancers in the year 2002. Int J Cancer 118: 3030-3044, 2006.

6. Petraccia L, Onori P, Sferra R, et al: MDR (multidrug resistance) in hepatocarcinoma clinical-therapeutic implications. Clin Ter 154: 325-335, 2003 (In Italian).

7. Zhang C, Liu L, Yu Y, Chen B, Tang C and Li X: Antitumor effects of ginsenoside $\mathrm{Rg} 3$ on human hepatocellular carcinoma cells. Mol Med Rep 5: 1295-1298, 2012.

8. Hardie DG, Carling D and Carlson M: The AMP-activated/SNF1 protein kinase subfamily: metabolic sensors of the eukaryotic cell. Annu Rev Biochem 67: 821-855, 1998

9. Hardie DG: Minireview: the AMP-activated protein kinase cascade: the key sensor of cellular energy status. Endocrinology 144: 5179-5183, 2003.

10. Hardie DG and Sakamoto K: AMPK: a key sensor of fuel and energy status in skeletal muscle. Physiology 21: 48-60, 2006.

11. Garcia-Gil M, Pesi R, Perna S, et al: 5'-Aminoimidazole4-carboxamide riboside induces apoptosis in human neuroblastoma cells. Neuroscience 117: 811-820, 2003.

12. Akhter MH, Sabir M and Bhide NK: Anti-inflammatory effect of berberine in rats injected locally with cholera toxin. Indian J Med Res 65: 133-141, 1977.

13. Hwang JM, Wang CJ, Chou FP, Tseng TH, Hsieh YS, Lin WL and Chu CY: Inhibitory effect of berberine on tert-butyl hydroperoxide-induced oxidative damage in rat liver. Arch Toxicol 76: 664-670, 2002

14. Tsai CS and Ochillo RF: Pharmacological effects of berberine on the longitudinal muscle of the guinea-pig isolated ileum. Arch Int Pharmacodyn Ther 310: 116-131, 1991.

15. Wang GY, Lv QH, Dong Q, Xu RZ and Dong QH: Berbamine induces Fas-mediated apoptosis in human hepatocellular carcinoma HepG2 cells and inhibits its tumor growth in nude mice. J Asian Nat Prod Res 11: 219-228, 2009.

16. Wang N, Feng Y, Zhu M, et al: Berberine induces autophagic cell death and mitochondrial apoptosis in liver cancer cells: the cellular mechanism. J Cell Biochem 111: 1426-1436, 2010.

17. Patil JB, Kim J and Jayaprakasha GK: Berberine induces apoptosis in breast cancer cells (MCF-7) through mitochondrial-dependent pathway. Eur J Pharmacol 645: 70-78, 2010.

18. Ho YT, Lu CC, Yang JS, et al: Berberine induced apoptosis via promoting the expression of caspase-8, -9 and -3, apoptosis-inducing factor and endonuclease $\mathrm{G}$ in SCC-4 human tongue squamous carcinoma cancer cells. Anticancer Res 29: 4063-4070, 2009.

19. Vichai V and Kirtikara K: Sulforhodamine B colorimetric assay for cytotoxicity screening. Nat Protoc 1: 1112-1116, 2006. 
20. Kim YM, Hwang JT, Kwak DW, et al: Involvement of AMPK signaling cascade in capsaicin-induced apoptosis of HT-29 colon cancer cells. Ann N Y Acad Sci 1095: 496-503, 2007.

21. Ji CB, Yang B, Yang YL, et al: Exogenous cell-permeable C6 ceramide sensitizes multiple cancer cell lines to Doxorubicininduced apoptosis by promoting AMPK activation and mTORC1 inhibition. Oncogene 29: 6557-6568, 2010.

22. Dorai T and Aggarwal BB: Role of chemopreventive agents in cancer therapy. Cancer Lett 5: 129-140, 2004.

23. Hwang JM, Kuo HC, Tseng TH, Liu JY and Chu CY: Berberine induces apoptosis through a mitochondria/caspases pathway in human hepatoma cells. Arch Toxicol 80: 62-73, 2006.

24. Hur JM, Hyun MS, Lim SY, Lee WY and Kim D: The combination of berberine and irradiation enhances anti-cancer effects via activation of p38 MAPK pathway and ROS generation in human hepatoma cells. J Cell Biochem 107: 955-964, 2009.
25. Liu B, Wang G, Yang J, Pan X, Yang Z and Zang L: Berberine inhibits human hepatoma cell invasion without cytotoxicity in healthy hepatocytes. PLoS One 6: e21416, 2011

26. Chao DC, Lin LJ, Kao ST, et al: Inhibitory effects of Zuo-Jin-Wan and its alkaloidal ingredients on activator protein 1 , nuclear factor- $\kappa \mathrm{B}$, and cellular transformation in HepG2 cells. Fitoterapia 82: 696-703, 2011.

27. Motoshima H, Goldstein BJ, Igata M and Araki E: AMPK and cell proliferation - AMPK as a therapeutic target for atherosclerosis and cancer. J Physiol 574: 63-71, 2006.

28. Luo Z, Saha AK, Xiang X and Ruderman NB: AMPK, the metabolic syndrome and cancer. Trends Pharmacol Sci 26: 69-76, 2005. 